\title{
UK Renal Registry 15th Annual Report: Chapter 9 Centre Variation in Access to Renal Transplantation in the UK (2006-2008)
}

\author{
Rishi Pruthi ${ }^{\mathrm{a}}$, Rommel Ravanan ${ }^{\mathrm{b}}$, John $\mathrm{O}^{\prime}$ Neill $^{\mathrm{c}}$, Paul Roderick ${ }^{\mathrm{d}}$, Laura Pankhurst ${ }^{\mathrm{c}}$, \\ Udaya Udayaraj ${ }^{b}$ \\ ${ }^{a}$ UK Renal Registry, Bristol, UK; ${ }^{\mathrm{b}}$ Southmead Hospital, Bristol, UK; ${ }^{\mathrm{C}}$ Organ Donation and Transplantation Directorate, \\ NHS Blood and Transplant, Bristol, UK; ${ }^{d}$ Southampton University, Southampton, UK
}

\section{Key Words}

Centre variation · Comorbidity · Donor after brainstem death - Donor after cardiac death · Equity of access · Living kidney donor - Outcomes · Patient factors · Quality improvement · Renal transplantation · Transplant waiting list

\begin{abstract}
Background: Renal transplantation is recognised as being the optimal treatment modality for many patients with established renal failure. This analysis aimed to explore inter-centre variation in access to renal transplantation in the UK. Methods: Transplant activity and waiting list data were obtained from NHS Blood and Transplant, demographic and laboratory data were obtained from the UK Renal Registry. All incident RRT patients starting treatment between 1st January 2006 and 31st December 2008 from
\end{abstract}

72 renal centres were considered for inclusion. The cohort was followed until 31st December 2010 (or until transplantation or death, whichever was earliest). Results: Age, ethnicity and primary renal diagnosis were associated with both accessing the kidney transplant waiting list and receiving a kidney transplant. A patient starting dialysis in a non-transplanting renal centre was less likely to be registered for transplantation (OR $0.80,95 \% \mathrm{Cl} 0.74-0.87$ ) or receive a transplant from a donor after cardiac death or a living kidney donor (OR 0.69, 95\% Cl 0.61-0.77) compared with patients cared for in transplanting renal centres. Once registered for kidney transplantation, patients in both transplanting and non-transplanting renal centres had an equal chance of receiving a transplant from a donor after brainstem death (OR 0.92, 95\% Cl 0.79-1.08). Conclusion: There was wide variation in access to kidney transplantation between UK renal centres which cannot be explained by differences in case mix.

$\begin{array}{ll}\text { KARGER } & \text { C 2013 S. Karger AG, Basel } \\ \text { Fax +4161306 1234 } & \text { 1160-2110/13/1235-0183\$38.00/0 } \\ \begin{array}{l}\text { E-Mail karger@karger.ch } \\ \text { www.karger.com }\end{array} & \begin{array}{l}\text { Accessible online at: } \\ \text { www.karger.com/nec }\end{array}\end{array}$

Email: renalregistry@renalregistry.nhs.uk 


\section{Introduction}

In an era where demand is increasingly outstripping supply, ensuring equity in access and allocation of a scarce resource that is a renal transplant poses many ethical and pragmatic dilemmas. For 'suitable' patients with established renal failure, renal transplantation confers both better quality of life and life expectancy than dialysis [1-3] and is the preferred modality of renal replacement therapy. Defining 'suitable' is a complex concept for which a series of national and international guidelines exist but most such guidelines do not have a robust evidence base for their recommendations. Therefore the fitness for transplantation assessment process ultimately revolves around conducting an individualised assessment of the risks of transplantation as well as the likely benefit. Centre practices and policies play an integral role in influencing this, although other patient specific factors are also known to influence access including age, gender, ethnicity, comorbidity and social deprivation [4-9].

In addition to influencing access to transplantation, centre practices and policies may also influence the likelihood of a patient receiving a living kidney donor or donor after cardiac death particularly during the time period this study covers, when the retrieving centre had the major influence on the distribution of such organs. Once a patient was on the waiting list, the probability of receiving a transplant from a donor after brainstem death however, was predominantly under the influence of the national organ allocation algorithm.

Achieving prompt and timely activation on the waiting list is important not least because increasing length of time on dialysis adversely affects graft and patient survival, but also because the current organ allocation algorithm introduced in April 2006 takes time spent on the waiting list into account when allocating deceased donor kidneys in the UK [10]. Thus, centres that achieve earlier listing for transplantation provide an advantage for their patients compared with centres that take longer.

This analysis aims to evaluate whether equity of access to the renal transplant list exists for patients with end stage renal disease across the UK, whether centres differ in the time taken to activate suitable patients on the waiting list and whether equity exists in the receipt of a renal transplant once the patient is on the transplant list (that is, the conversion efficiency from being on the waiting list to receiving a transplant). Patient specific and independent variables that influenced access to the waiting list or transplantation were analysed.

\section{Methods}

\section{Study population}

All adult patients starting renal replacement therapy $(\mathrm{n}=19,780)$ between 1st January 2006 and 31st December 2008 in renal centres $(n=72)$ returning data to the UK Renal Registry (UKRR) were considered for inclusion. For the analysis of the proportion of a centre's patients included on the waiting list, patients aged 65 years or above $(n=9,636)$, patients with inappropriate activation and early suspension as described below $(\mathrm{n}=146)$ and patients listed for multi-organ transplants other than pancreas $(n=37)$ were excluded, resulting in a final cohort of 9,961 patients. These patients were followed to 31st December 2010 or until they were put on the waiting list for kidney transplant alone, kidney plus pancreas transplant, or death, whichever was earliest. For the analysis of the proportion transplanted, all patients from the incident cohort who were activated on the waiting list before 31st December 2009 $(\mathrm{n}=5,755)$ were followed until 31st December 2011, to estimate the proportion transplanted with a kidney alone or kidney plus pancreas within two years of inclusion on the waiting list.

\section{Exclusions}

Patients listed for multi-organ transplants other than pancreas were excluded as were those who were suspended for more than 30 days within 90 days of first activation. The latter avoided any potential bias from centres that may activate patients on the transplant list and then immediately suspend them before more permanent activation at a later date after more formal medical assessment of the patient's fitness.

\section{Data analysed}

Information on start date of renal replacement therapy and relevant patient level data including age (grouped as 18-29, 30-39, 40-49, 50-59, 60-64), gender, ethnicity (white, nonWhite, missing) and PRD (primary renal diagnosis classified as: patient with diabetes, patient without diabetes, missing) came from the UKRR. The date of activation on the kidney transplant waiting list, date of transplantation, or both came from the UK Transplant Registry held by the Organ Donation and Transplantation Directorate of NHS Blood and Transplant.

\section{Statistical methods}

A logistic regression model was developed to identify the influence of patient specific variables including age, gender, ethnicity and primary renal diagnosis, on the probability of access to the transplant list and receipt of a transplant once on the waiting list. After adjusting for patient specific variables, the percentage of patients activated on the transplant list and the percentage of patients on the waiting list who achieved a transplant in each centre were determined. The overall effect of the centre associated with each analysis was assessed by including renal centre as a random effect in the risk-adjusted logistic regression model. The extent of variation between centres was determined by using a log likelihood ratio test that provided the change in the value of $-2 \operatorname{LogL}$ on inclusion of the random centre effect. SAS 9.3 was used for analyses; a p value of less than $5 \%$ was considered significant.

To analyse access to the transplant list, the proportion of incident patients with end stage renal disease in each centre 
who were subsequently activated on the waiting list within two years of starting renal replacement therapy was identified. All patients who achieved live donor transplantation without prior activation on the national transplant waiting list were assumed to have been activated for the purposes of this analysis. Time to activation on the waiting list was defined as the interval between the start of RRT and the date of activation on the waiting list. Patients achieving pre-emptive deceased donor transplantation were considered to have been activated on the same day as starting RRT i.e. a time to activation of 0 days. Patients achieving preemptive live donor transplantation without prior activation on the national transplant list were considered to have been 'active' on the list for an arbitrary time of six months. This was to take into account an average of six months required by most centres to complete live donor fitness evaluation and hence the likelihood that the intended recipient was considered fit for transplantation (and by inference suitable to be active on the waiting list) for that duration. This was done to account for different centre practices with regard to listing patients on the deceased donor list prior to receiving a living donor transplant.

The median time to activation was estimated from the KaplanMeier plot for patients at each renal centre, with the event as the date of activation and censoring at death or on 31st December 2010, whichever was earlier. Data from patients who did not achieve activation were included in the calculation of median times using this method, thus providing a meaningful estimate of the true time to activation. Including only those patients activated would produce a biased estimate. The overall centre effect associated with time to activation was calculated by including renal centre as a variable in a risk- adjusted Cox regression model.

To analyse the differences between centres in achieving a renal transplant, the percentage of patients activated on the waiting list who received a renal transplant within two years of being activated was estimated (conversion efficiency). The conversion efficiency for receiving a transplant from a donor after brainstem death or a donor after cardiac death/living kidney donor were analysed separately. Receipt of a kidney from a donor after brainstem death was predominantly influenced by national allocation policy, whereas receipt from a donor after cardiac death/live donor kidney was much more dependent on local transplant centre practices. For the cohort under consideration, donor after cardiac death transplantation was predominantly a locally managed service.

Funnel plots are used to present the results for each outcome of interest, providing a visual comparison of each centre's performance compared with its peers. Where relevant, the funnel plots are adjusted for patient specific variables influencing that outcome. The solid black straight line in each funnel plot shows the overall average together with the 95\% and $99.8 \%$ confidence intervals, which correspond to two and three standard deviations from the mean. Each point on the plot represents one renal centre. With 72 centres included, for each outcome of interest, three centres would be predicted to fall between the $95 \%$ and $99.8 \%$ confidence intervals and no centre should fall outside the $99.8 \%$ confidence interval. Centres $(\mathrm{n}=3)$ with fewer than 10 patients activated on the waiting list are not included in the funnel plots.

The analysis methodology described above is identical to a previous independent peer reviewed publication [11].

\section{Results}

The results of the logistic regression model analysis of patient characteristics influencing access to the waiting list are presented in table 9.1. Ethnicity data were missing for $17.1 \%$ of patients and PRD for $5.3 \%$ of patients.

Tables 9.2 and 9.3 show the results of the logistic regression analysis of factors influencing the likelihood of receiving a transplant from a donor after brainstem death and the analysis of factors influencing receipt of a transplant from a donor after cardiac death or a

Table 9.1. Patient factors influencing activation on the national kidney transplant waiting list within two years of RRT start

\begin{tabular}{|c|c|c|c|c|c|}
\hline Factor & $\begin{array}{c}\text { Category } \\
\text { (at baseline) }\end{array}$ & $\begin{array}{l}\text { Patients } \\
\text { N }(\%)\end{array}$ & $\begin{array}{l}\text { Odds } \\
\text { ratio }\end{array}$ & $95 \% \mathrm{CI}$ & $\mathrm{P}$ value \\
\hline Age & $\begin{array}{c}(18-29) \\
30-39 \\
40-49 \\
50-59 \\
60-64\end{array}$ & $\begin{aligned} 898 & (9.0) \\
1,442 & (14.5) \\
2,378 & (23.9) \\
3,171 & (31.8) \\
2,072 & (20.8)\end{aligned}$ & $\begin{array}{l}1.00 \\
0.78 \\
0.51 \\
0.26 \\
0.13\end{array}$ & $\begin{array}{c}\text { ref } \\
0.63-0.96 \\
0.42-0.62 \\
0.21-0.31 \\
0.11-0.16\end{array}$ & $\begin{aligned} & \mathrm{n} / \mathrm{a} \\
& 0.02 \\
< & 0.0001 \\
< & 0.0001 \\
< & 0.0001\end{aligned}$ \\
\hline Gender & $\begin{array}{l}\text { (Male) } \\
\text { Female }\end{array}$ & $\begin{array}{l}6,057(60.8) \\
3,904(39.2)\end{array}$ & $\begin{array}{l}1.00 \\
0.92\end{array}$ & $\begin{array}{c}\text { ref } \\
0.84-1.00\end{array}$ & $\begin{array}{r}\mathrm{n} / \mathrm{a} \\
0.05\end{array}$ \\
\hline PRD & $\begin{array}{c}\text { (Non-diabetic) } \\
\text { Diabetic } \\
\text { Missing }\end{array}$ & $\begin{array}{c}7,096(71.2) \\
2,335(23.4) \\
530(5.3)\end{array}$ & $\begin{array}{l}1.00 \\
0.43 \\
0.65\end{array}$ & $\begin{array}{c}\text { ref } \\
0.39-0.48 \\
0.54-0.79\end{array}$ & $\begin{array}{c}\mathrm{n} / \mathrm{a} \\
<0.0001 \\
<0.0001\end{array}$ \\
\hline
\end{tabular}

ref - reference category, n/a - not applicable 
Table 9.2. Patient factors affecting the probability of receiving a transplant from a donor after brainstem death within two years of registration on the national kidney transplant waiting list

\begin{tabular}{|c|c|c|c|c|c|}
\hline Factor & $\begin{array}{c}\text { Category } \\
\text { (at baseline) }\end{array}$ & $\begin{array}{l}\text { Patients } \\
\mathrm{N}(\%)\end{array}$ & $\begin{array}{l}\text { Odds } \\
\text { ratio }\end{array}$ & $95 \% \mathrm{CI}$ & $\mathrm{P}$ value \\
\hline Age & $\begin{array}{c}(18-29) \\
30-39 \\
40-49 \\
50-59 \\
60-64\end{array}$ & $\begin{array}{r}731(12.7) \\
1,089(18.9) \\
1,603(27.9) \\
1,599(27.8) \\
733(12.7)\end{array}$ & $\begin{array}{l}1.00 \\
1.20 \\
0.76 \\
0.35 \\
0.17\end{array}$ & $\begin{array}{c}\text { ref } \\
0.94-1.53 \\
0.60-0.96 \\
0.27-0.45 \\
0.12-0.25\end{array}$ & $\begin{array}{c}\mathrm{n} / \mathrm{a} \\
0.14 \\
0.02 \\
<0.0001 \\
<0.0001\end{array}$ \\
\hline Ethnicity & $\begin{array}{c}\text { (White) } \\
\text { Non-White } \\
\text { Missing }\end{array}$ & $\begin{array}{r}3,829(66.5) \\
1,145(19.9) \\
781(13.6)\end{array}$ & $\begin{array}{l}1.00 \\
0.50 \\
0.84\end{array}$ & $\begin{array}{c}\text { ref } \\
0.41-0.63 \\
0.67-1.06\end{array}$ & $\begin{array}{c}\mathrm{n} / \mathrm{a} \\
<0.0001 \\
0.14\end{array}$ \\
\hline Gender & $\begin{array}{l}\text { (Male) } \\
\text { Female }\end{array}$ & $\begin{array}{l}3,528(61.3) \\
2,227(38.7)\end{array}$ & $\begin{array}{l}1.00 \\
0.93\end{array}$ & $\begin{array}{c}\text { ref } \\
0.80-1.09\end{array}$ & $\begin{array}{r}\mathrm{n} / \mathrm{a} \\
0.38\end{array}$ \\
\hline PRD & $\begin{array}{c}\text { (Non-diabetic) } \\
\text { Diabetic } \\
\text { Missing }\end{array}$ & $\begin{array}{c}4,501(78.2) \\
971(16.9) \\
283(4.9)\end{array}$ & $\begin{array}{l}1.00 \\
5.03 \\
1.17\end{array}$ & $\begin{array}{c}\text { ref } \\
4.24-5.96 \\
0.81-1.69\end{array}$ & $\begin{array}{c}\mathrm{n} / \mathrm{a} \\
<0.0001 \\
0.4\end{array}$ \\
\hline
\end{tabular}

ref - reference category, n/a - not applicable

living kidney donor. Ethnicity data were missing for $13.6 \%$ of patients and PRD for $4.9 \%$ of patients.

A patient starting dialysis in a non-transplanting renal centre was less likely to be registered for transplantation (OR $0.80,95 \%$ CI $0.74-0.87$ ) or receive a transplant from a donor after cardiac death or a living kidney donor (OR 0.69, 95\% CI 0.61-0.77) compared with patients managed in transplanting renal centres. Once registered for kidney transplantation, patients in both transplant- ing and non-transplanting renal centres had an equal chance of receiving a transplant from a donor after brainstem death (OR 0.92, 95\% CI 0.79-1.08).

After adjusting for patient specific variables that were shown to influence outcome (age, ethnicity, gender, PRD), significant centre effects were identified for the probability of being activated on the waiting list (figure 9.1 and table 9.4) (change in $-2 \log L=264.4$, $\mathrm{df}($ degrees of freedom $)=1, \mathrm{p}<0.0001)$.

Table 9.3. Patient factors affecting the probability of receiving a transplant from a donor after cardiac death or living kidney donor within two years of registration on the national kidney transplant waiting list

\begin{tabular}{|c|c|c|c|c|c|}
\hline Factor & $\begin{array}{c}\text { Category } \\
\text { (at baseline) }\end{array}$ & $\begin{array}{l}\text { Patients } \\
\text { N }(\%)\end{array}$ & $\begin{array}{l}\text { Odds } \\
\text { ratio }\end{array}$ & $95 \% \mathrm{CI}$ & $\mathrm{P}$ value \\
\hline Age & $\begin{array}{c}(18-29) \\
30-39 \\
40-49 \\
50-59 \\
60-64\end{array}$ & $\begin{array}{r}731(12.7) \\
1,089(18.9) \\
1,603(27.9) \\
1,599(27.8) \\
733(12.7)\end{array}$ & $\begin{array}{l}1.00 \\
0.63 \\
0.59 \\
0.40 \\
0.41\end{array}$ & $\begin{array}{c}\text { ref } \\
0.52-0.77 \\
0.49-0.71 \\
0.34-0.49 \\
0.33-0.52\end{array}$ & $\begin{aligned} & \mathrm{n} / \mathrm{a} \\
< & 0.0001 \\
< & 0.0001 \\
< & 0.0001 \\
< & 0.0001\end{aligned}$ \\
\hline Ethnicity & $\begin{array}{l}\text { (White) } \\
\text { Non-White } \\
\text { Missing }\end{array}$ & $\begin{array}{r}3,829(66.5) \\
1,145(19.9) \\
781(13.6)\end{array}$ & $\begin{array}{l}1.00 \\
0.48 \\
0.63\end{array}$ & $\begin{array}{c}\text { ref } \\
0.41-0.56 \\
0.53-0.75\end{array}$ & $\begin{array}{c}\mathrm{n} / \mathrm{a} \\
<0.0001 \\
<0.0001\end{array}$ \\
\hline Gender & $\begin{array}{l}\text { (Male) } \\
\text { Female }\end{array}$ & $\begin{array}{l}3,528(61.3) \\
2,227(38.7)\end{array}$ & $\begin{array}{l}1.00 \\
0.91\end{array}$ & $\begin{array}{c}\text { ref } \\
0.81-1.02\end{array}$ & $\begin{array}{r}\mathrm{n} / \mathrm{a} \\
0.11\end{array}$ \\
\hline PRD & $\begin{array}{c}\text { (Non-diabetic) } \\
\text { Diabetic } \\
\text { Missing }\end{array}$ & $\begin{array}{cc}4,501 & (78.2) \\
971 & (16.9) \\
283 & (4.9)\end{array}$ & $\begin{array}{l}1.00 \\
0.33 \\
1.09\end{array}$ & $\begin{array}{c}\text { ref } \\
0.28-0.40 \\
0.85-1.40\end{array}$ & $\begin{array}{c}\mathrm{n} / \mathrm{a} \\
<0.0001 \\
0.5\end{array}$ \\
\hline
\end{tabular}

ref - reference category, n/a - not applicable 


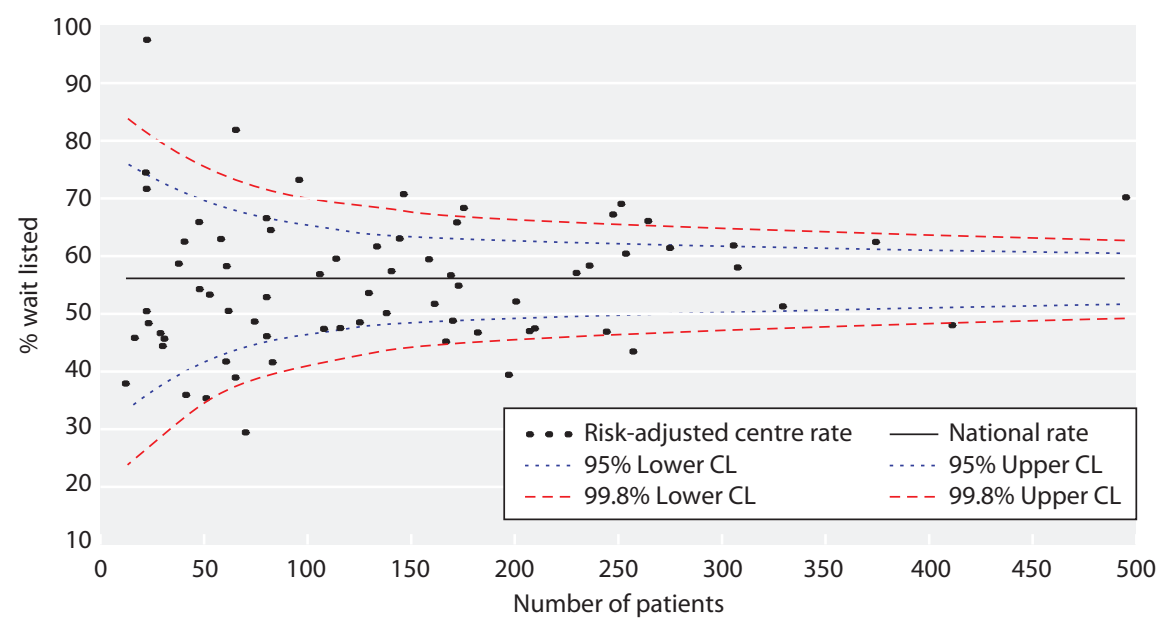

Fig. 9.1. The percentage of patients wait listed for a kidney transplant by renal centre, prior to or within two years of starting dialysis (centres with $<10$ patients excluded)

Table 9.4. The percentage of patients wait listed for a kidney transplant by renal centre, prior to or within two years of starting dialysis

\begin{tabular}{|c|c|c|c|c|c|c|c|c|c|}
\hline \multirow[b]{2}{*}{ Centre } & \multirow{2}{*}{$\begin{array}{l}\text { RRT } \\
\mathrm{N}\end{array}$} & \multirow{2}{*}{$\begin{array}{c}\text { Registrations } \\
\mathrm{N}\end{array}$} & \multicolumn{2}{|c|}{$\%$ wait listed } & \multirow[b]{2}{*}{ Centre } & \multirow{2}{*}{$\begin{array}{l}\text { RRT } \\
\mathrm{N}\end{array}$} & \multirow{2}{*}{$\begin{array}{c}\text { Registrations } \\
\text { N }\end{array}$} & \multicolumn{2}{|c|}{$\%$ wait listed } \\
\hline & & & Unadjusted & Risk-adjusted & & & & Unadjusted & Risk-adjusted \\
\hline Airdrie & 80 & 39 & 48.8 & 53.0 & L Guys & 307 & 193 & 62.9 & 61.8 \\
\hline Antrim & 30 & 12 & 40.0 & 44.2 & L Kings & 208 & 100 & 48.1 & 46.9 \\
\hline B Heart & 138 & 70 & 50.7 & 50.2 & L Rfree & 308 & 187 & 60.7 & 58.2 \\
\hline Basldn & 61 & 26 & 42.6 & 41.4 & Leeds & 237 & 144 & 60.8 & 58.3 \\
\hline Belfast & 129 & 73 & 56.6 & 53.7 & Leic & 375 & 244 & 65.1 & 62.6 \\
\hline Bradfd & 115 & 56 & 48.7 & 47.4 & Liv Ain & 51 & 16 & 31.4 & 34.3 \\
\hline Brightn & 144 & 88 & 61.1 & 63.0 & Liv RI & 201 & 110 & 54.7 & 52.3 \\
\hline Bristol & 254 & 157 & 61.8 & 60.6 & M RI & 169 & 101 & 59.8 & 56.6 \\
\hline Camb & 197 & 84 & 42.6 & 39.6 & Middlbr & 134 & 86 & 64.2 & 62.1 \\
\hline Clwyd & 23 & 9 & 39.1 & 48.1 & Oxford & 247 & 171 & 69.2 & 67.3 \\
\hline Colchr & 22 & 12 & 54.5 & 71.8 & Plymth & 95 & 69 & 72.6 & 73.5 \\
\hline Covnt & 170 & 86 & 50.6 & 48.6 & Ports & 252 & 181 & 71.8 & 69.0 \\
\hline D \& Gall & 22 & 16 & 72.7 & 97.7 & Prestn & 210 & 100 & 47.6 & 47.5 \\
\hline Derby & 113 & 65 & 57.5 & 59.5 & Redng & 147 & 108 & 73.5 & 70.7 \\
\hline Derry & 12 & 4 & 33.3 & 38.0 & Salford & 230 & 137 & 59.6 & 56.9 \\
\hline Donc & 22 & 16 & 72.7 & 74.7 & Sheff & 245 & 118 & 48.2 & 46.8 \\
\hline Dorset & 80 & 55 & 68.8 & 66.5 & Shrew & 83 & 36 & 43.4 & 41.4 \\
\hline Dudley & 70 & 21 & 30.0 & 29.4 & Stevng & 167 & 76 & 45.5 & 43.9 \\
\hline Dundee & 61 & 29 & 47.5 & 58.3 & Sthend & 58 & 34 & 58.6 & 63.1 \\
\hline Dunfn & 48 & 26 & 54.2 & 65.9 & Stoke & 75 & 37 & 49.3 & 48.6 \\
\hline Klmarnk & 65 & 19 & 29.2 & 38.9 & York & 48 & 28 & 58.3 & 54.2 \\
\hline
\end{tabular}




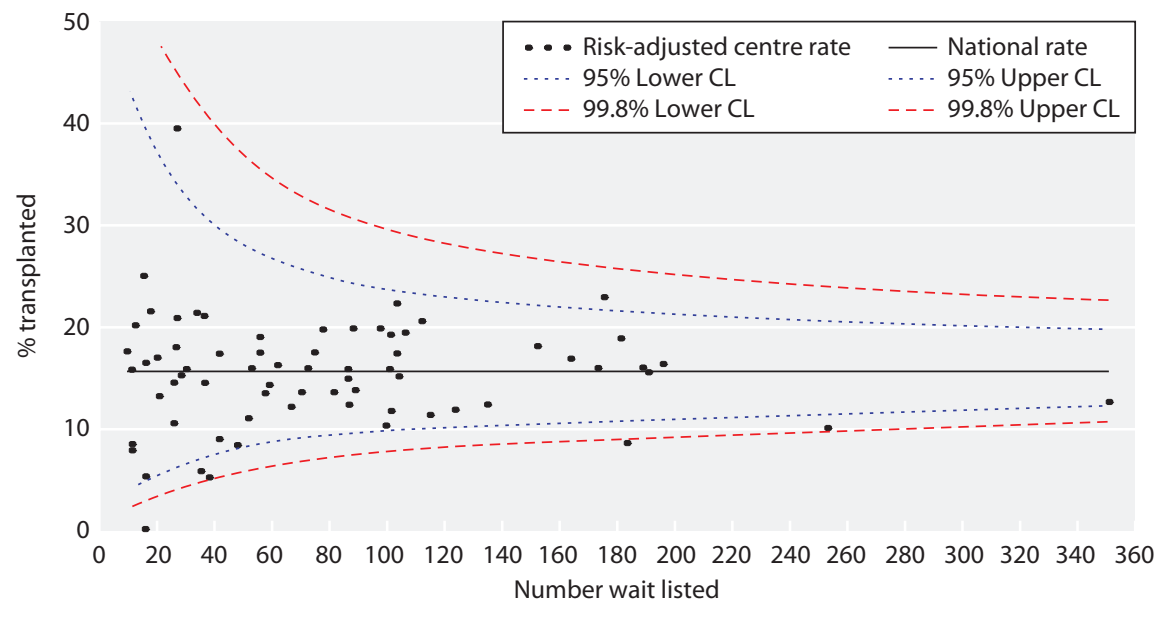

Fig. 9.2. The percentage of patients receiving a transplant from a donor after brainstem death by renal centre, within two years of transplant waiting list registration (centres with $<10$ patients excluded)
After adjustment for patient variables, significant centre differences were seen in the probability of receiving a renal transplant from a donor after brainstem death (figure 9.2 and table 9.5) (change in $-2 \log \mathrm{L}=6.0, \mathrm{df}=1, \mathrm{p}=0.015$ ) or a donor after cardiac death/living kidney donor (figure 9.3 and table 9.5) (change in $-2 \log \mathrm{L}=172.9, \mathrm{df}=1, \mathrm{p}<0.0001$ ). As shown, several centres fall outside the $95 \%$ and $99.8 \%$ confidence intervals.

Figure 9.4 and table 9.6 show the unadjusted median time taken to activate patients on the transplant list for each renal centre.

The funnel plot is based on the assumption of an exponential distribution for time to activation. Although this assumption is broadly consistent with the data, the model based estimate of the national median was greater than that observed. This leads to an unusually large number of centres falling outside the lower $99.8 \%$ confidence limit for this national rate and perhaps too few occurring outside the upper limit. However, the plot highlights those centres that have significantly longer time to activation but small numbers of patients on the waiting list. The Cox model giving a riskadjusted analysis of time to activation identified a significant effect of centre variation (change in $-2 \log \mathrm{L}=458.0, \mathrm{df}=71, \mathrm{p}<0.0001) . \quad$ In general, centres with the longest unadjusted waiting times also had the longest risk-adjusted waiting times. The four centres lying outside the upper $99.8 \%$ confidence limit all had hazard ratios that indicated a significant delay in the chance of wait listing compared with a baseline centre that had a median time comparable to the national median.

\section{Discussion}

\section{Patient level factors affecting access}

The observation that increasing age was seen to be negatively associated with access to transplantation was not unexpected as the risk-benefit ratio of receiving a renal transplant alters with age. Increased comorbidity burden in older patients may require more intensive time consuming investigations prior to listing and may also deem them unsuitable in some cases. Interestingly, whilst previous reports [12] have cited female gender to be associated with a reduced likelihood to receive a kidney after brainstem death, this was not noted in this study.

Ethnicity has sometimes been cited as being a cause of inequity in accessing transplantation, although it was reassuring to see that in this study it was not seen to impact a patients' probability of being listed (consistent with earlier work undertaken by Udayaraj and colleagues) [13]. It was however seen to be negatively associated with receiving a kidney once listed from a living kidney donor, donor after brainstem death or donor after cardiac death. A likely cause for this may be the widely acknowledged lack of donors from ethnic minorities contributing to the donor pool, as well as the importance given to HLA matching in the national allocation protocol which may have favoured a predominantly white donor pool being matched with white recipients. Although the allocation protocol changed in April 2006 (during the study period) the lack of an observed impact may be due to the fact that all patients in this study irrespective of ethnicity were likely to have been on the waiting list for a similar duration of time, 
Table 9.5. The percentage of patients receiving a transplant, by donor type and renal centre, within two years of transplant waiting list registration

\begin{tabular}{|c|c|c|c|c|c|c|c|}
\hline \multirow{2}{*}{ Centre } & \multirow{2}{*}{$\begin{array}{l}\text { Listed } \\
\mathrm{N}\end{array}$} & \multicolumn{3}{|c|}{ Organ from donor after brainstem death } & \multicolumn{3}{|c|}{ Organ from living kidney donor/donor after cardiac death } \\
\hline & & $\begin{array}{c}\text { Transplanted } \\
\text { N }\end{array}$ & \multicolumn{2}{|c|}{ Transplant rate $(\%)$} & $\begin{array}{c}\text { Transplanted } \\
\text { N }\end{array}$ & \multicolumn{2}{|c|}{ Transplant rate $(\%)$} \\
\hline Abrdn & 52 & 5 & 9.6 & 10.9 & 6 & 11.5 & 13.6 \\
\hline Airdrie & 42 & 11 & 26.2 & 17.3 & 7 & 16.7 & 16.9 \\
\hline B QEH & 184 & 14 & 7.6 & 8.5 & 50 & 27.2 & 28.7 \\
\hline Bangor & 13 & 4 & 30.8 & 20.0 & 2 & 15.4 & 13.4 \\
\hline Basldn & 27 & 6 & 22.2 & 18.0 & 12 & 44.4 & 40.1 \\
\hline Belfast & 75 & 13 & 17.3 & 17.4 & 14 & 18.7 & 15.6 \\
\hline Bradfd & 56 & 9 & 16.1 & 17.4 & 15 & 26.8 & 26.5 \\
\hline Carlis & 27 & 9 & 33.3 & 39.4 & 9 & 33.3 & 28.6 \\
\hline Carsh & 115 & 12 & 10.4 & 11.2 & 37 & 32.2 & 32.9 \\
\hline Chelms & 26 & 2 & 7.7 & 10.4 & 8 & 30.8 & 28.9 \\
\hline Clwyd & 10 & 2 & 20.0 & 17.4 & 3 & 30.0 & 27.9 \\
\hline Colchr & 12 & 1 & 8.3 & 8.5 & 6 & 50.0 & 54.7 \\
\hline Covnt & 87 & 13 & 14.9 & 14.9 & 40 & 46.0 & 42.6 \\
\hline D \& Gall & 16 & 2 & 12.5 & 16.3 & 7 & 43.8 & 54.2 \\
\hline Derby & 67 & 8 & 11.9 & 12.1 & 6 & 9.0 & 8.9 \\
\hline Derry & 4 & 1 & 25.0 & 47.3 & 0 & 0.0 & 0.0 \\
\hline Donc & 16 & 1 & 6.3 & 5.0 & 1 & 6.3 & 6.0 \\
\hline Dorset & 56 & 12 & 21.4 & 18.9 & 13 & 23.2 & 22.5 \\
\hline Dudley & 26 & 4 & 15.4 & 14.6 & 3 & 11.5 & 10.5 \\
\hline Ipswi & 34 & 7 & 20.6 & 21.3 & 15 & 44.1 & 38.1 \\
\hline Kent & 100 & 10 & 10.0 & 10.2 & 44 & 44.0 & 39.8 \\
\hline Klmarnk & 21 & 3 & 14.3 & 13.2 & 3 & 14.3 & 17.6 \\
\hline L Barts & 197 & 27 & 13.7 & 16.2 & 72 & 36.5 & 41.4 \\
\hline L Guys & 192 & 33 & 17.2 & 15.5 & 105 & 54.7 & 58.5 \\
\hline L Kings & 102 & 10 & 9.8 & 11.6 & 23 & 22.5 & 25.5 \\
\hline L Rfree & 190 & 26 & 13.7 & 16.0 & 59 & 31.1 & 32.1 \\
\hline L St.G & 62 & 8 & 12.9 & 16.2 & 34 & 54.8 & 53.5 \\
\hline L West & 351 & 37 & 10.5 & 12.6 & 156 & 44.4 & 54.7 \\
\hline Leeds & 153 & 25 & 16.3 & 18.0 & 64 & 41.8 & 39.6 \\
\hline Leic & 254 & 23 & 9.1 & 9.9 & 88 & 34.6 & 33.8 \\
\hline Liv Ain & 15 & 3 & 20.0 & 24.9 & 4 & 26.7 & 24.5 \\
\hline Liv RI & 113 & 24 & 21.2 & 20.4 & 40 & 35.4 & 32.2 \\
\hline M RI & 104 & 25 & 24.0 & 22.2 & 28 & 26.9 & 26.1 \\
\hline Middlbr & 88 & 21 & 23.9 & 19.8 & 35 & 39.8 & 39.5 \\
\hline Newc & 102 & 21 & 20.6 & 19.1 & 44 & 43.1 & 41.2 \\
\hline Newry & 12 & 2 & 16.7 & 15.6 & 0 & 0.0 & 0.0 \\
\hline Norwch & 59 & 9 & 15.3 & 14.1 & 21 & 35.6 & 34.1 \\
\hline
\end{tabular}


Table 9.5. Continued

\begin{tabular}{|c|c|c|c|c|c|c|c|}
\hline \multirow{2}{*}{ Centre } & \multirow{2}{*}{$\begin{array}{l}\text { Listed } \\
\mathrm{N}\end{array}$} & \multicolumn{3}{|c|}{ Organ from donor after brainstem death } & \multicolumn{3}{|c|}{ Organ from living kidney donor/donor after cardiac death } \\
\hline & & $\begin{array}{c}\text { Transplanted } \\
\text { N }\end{array}$ & \multicolumn{2}{|c|}{ Transplant rate $(\%)$} & $\begin{array}{c}\text { Transplanted } \\
\text { N }\end{array}$ & \multicolumn{2}{|c|}{ Transplant rate $(\%)$} \\
\hline Nottm & 104 & 20 & 19.2 & 17.4 & 29 & 27.9 & 26.8 \\
\hline Oxford & 176 & 50 & 28.4 & 22.8 & 52 & 29.5 & 30.1 \\
\hline Prestn & 105 & 14 & 13.3 & 15.1 & 30 & 28.6 & 26.7 \\
\hline Redng & 107 & 21 & 19.6 & 19.3 & 38 & 35.5 & 37.2 \\
\hline Salford & 135 & 15 & 11.1 & 12.3 & 38 & 28.1 & 26.2 \\
\hline Sheff & 124 & 14 & 11.3 & 11.9 & 43 & 34.7 & 30.8 \\
\hline Shrew & 38 & 2 & 5.3 & 5.1 & 11 & 28.9 & 25.0 \\
\hline Swanse & 82 & 12 & 14.6 & 13.4 & 25 & 30.5 & 29.4 \\
\hline Truro & 48 & 4 & 8.3 & 8.3 & 30 & 62.5 & 65.2 \\
\hline Tyrone & 16 & 0 & 0.0 & 0.0 & 1 & 6.3 & 5.6 \\
\hline Ulster & 6 & 0 & 0.0 & 0.0 & 0 & 0.0 & 0.0 \\
\hline Wirral & 37 & 5 & 13.5 & 14.3 & 11 & 29.7 & 25.5 \\
\hline Wolve & 58 & 8 & 13.8 & 13.3 & 20 & 34.5 & 34.0 \\
\hline Wrexm & 18 & 6 & 33.3 & 21.4 & 6 & 33.3 & 29.9 \\
\hline York & 27 & 7 & 25.9 & 20.7 & 5 & 18.5 & 16.5 \\
\hline
\end{tabular}

whereas the new allocation policy would primarily have improved access for those listed well before 2006 (not included in this study).

Diabetes was also seen to affect wait listing adversely although this is not surprising as many would be subject to additional diabetic complications and increased cardiovascular risk that would need to be managed. The higher proportion of patients with diabetes receiving a transplant corresponds to an increase in the number of simultaneous kidney-pancreas transplants during the study period, as the allocation algorithm prioritised dual organ recipients.

When interpreting the analyses in this chapter it is important to consider the potential impact of missing data on the results. Missing data occurs as a result of either a renal centre failing to complete relevant fields

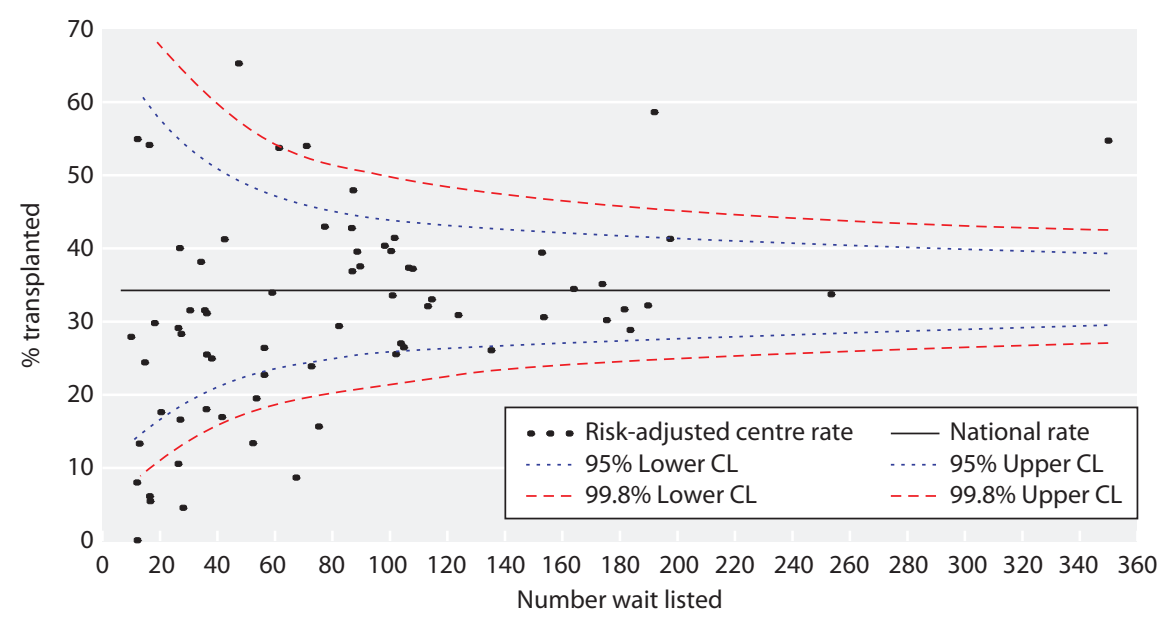

190
Fig. 9.3. The percentage of patients receiving a transplant from a living kidney donor/donor after cardiac death by renal centre, within two years of transplant waiting list registration (centres with $<10$ patients excluded) 


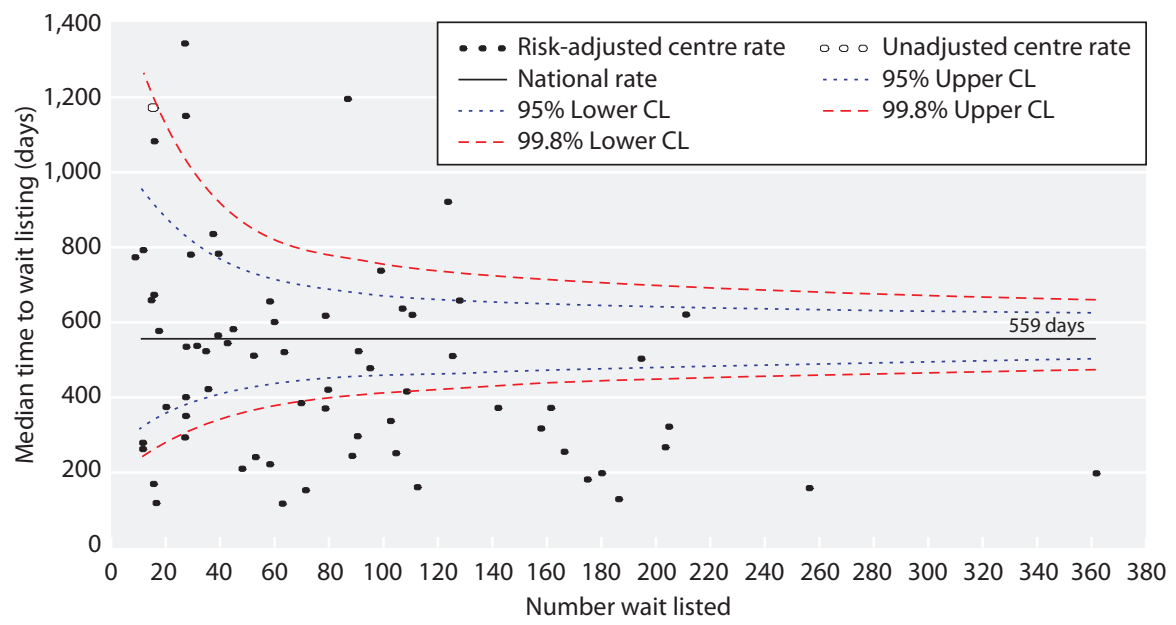

Fig. 9.4. Median time to wait listing for a kidney transplant, by renal centre (centres with $<10$ patients excluded)

The centre represented by an unfilled symbol has its final event time as the plotting position as the median time could not be estimated

Table 9.6. Median time to wait listing for a kidney transplant, by renal centre (censoring at the earliest of death or 31st December 2010)

\begin{tabular}{|c|c|c|c|c|c|c|c|}
\hline Centre & $\begin{array}{l}\text { RRT } \\
\mathrm{N}\end{array}$ & $\begin{array}{l}\text { Registrations } \\
\mathrm{N}\end{array}$ & $\begin{array}{l}\text { Median time to listing } \\
\text { (days) }\end{array}$ & Centre & $\begin{array}{l}\text { RRT } \\
\mathrm{N}\end{array}$ & $\begin{array}{l}\text { Registrations } \\
\mathrm{N}\end{array}$ & $\begin{array}{l}\text { Median time to listing } \\
\text { (days) }\end{array}$ \\
\hline Abrdn & 88 & 53 & 511 & L Barts & 411 & 212 & 623 \\
\hline Airdrie & 80 & 45 & 580 & L Guys & 307 & 204 & 269 \\
\hline Antrim & 30 & 13 & 794 & L Kings & 208 & 108 & 635 \\
\hline B Heart & 138 & 79 & 620 & L Rfree & 308 & 205 & 322 \\
\hline B QEH & 330 & 195 & 501 & L St.G & 106 & 64 & 120 \\
\hline Bangor & 41 & 16 & 1089 & L West & 496 & 362 & 202 \\
\hline Basldn & 61 & 30 & 786 & Leeds & 237 & 159 & 320 \\
\hline Belfast & 129 & 79 & 369 & Leic & 375 & 257 & 153 \\
\hline Bradfd & 115 & 60 & 603 & Liv Ain & 51 & 17 & $1181^{\star}$ \\
\hline Brightn & 144 & 91 & 296 & Liv RI & 201 & 126 & 512 \\
\hline Bristol & 254 & 167 & 255 & M RI & 169 & 109 & 418 \\
\hline Camb & 197 & 88 & 1197 & Middlbr & 134 & 89 & 245 \\
\hline Cardff & 276 & 176 & 180 & Newc & 173 & 103 & 341 \\
\hline Carlis & 40 & 27 & 293 & Newry & 22 & 12 & 262 \\
\hline Carsh & 258 & 124 & 926 & Norwch & 125 & 64 & 526 \\
\hline Chelms & 53 & 28 & 534 & Nottm & 183 & 111 & 623 \\
\hline Clwyd & 23 & 10 & 778 & Oxford & 247 & 181 & 197 \\
\hline Colchr & 22 & 12 & 280 & Plymth & 95 & 72 & 156 \\
\hline Covnt & 170 & 95 & 482 & Ports & 252 & 187 & 129 \\
\hline D \& Gall & 22 & 16 & 174 & Prestn & 210 & 111 & 622 \\
\hline Derby & 113 & 70 & 384 & Redng & 147 & 113 & 159 \\
\hline Derry & 12 & 5 & 881 & Salford & 230 & 142 & 376 \\
\hline Donc & 22 & 17 & 120 & Sheff & 245 & 129 & 663 \\
\hline Dorset & 80 & 59 & 223 & Shrew & 83 & 40 & 786 \\
\hline Dudley & 70 & 28 & 1155 & Stevng & 167 & 99 & 742 \\
\hline Dundee & 61 & 32 & 540 & Sthend & 58 & 36 & 420 \\
\hline Dunfn & 48 & 28 & 356 & Stoke & 75 & 40 & 567 \\
\hline Edinb & 172 & 102 & 338 & Sund & 79 & 43 & 549 \\
\hline Exeter & 141 & 80 & 419 & Swanse & 161 & 91 & 523 \\
\hline Glasgw & 265 & 162 & 373 & Truro & 65 & 49 & 213 \\
\hline Glouc & 82 & 54 & 242 & Tyrone & 29 & 16 & 667 \\
\hline Hull & 175 & 104 & 333 & Ulster & 16 & 7 & 786 \\
\hline Inverns & 37 & 21 & 371 & Wirral & 77 & 39 & 838 \\
\hline Ipswi & 61 & 35 & 519 & Wolve & 108 & 59 & 656 \\
\hline Kent & 158 & 105 & 252 & Wrexm & 30 & 18 & 579 \\
\hline Klmarnk & 65 & 28 & 1347 & York & 48 & 28 & 400 \\
\hline
\end{tabular}

${ }^{*}$ Results in bold italics are final event times as median times could not be estimated 
on their renal IT system or a failure to extract this data. Missing data may not be at random; sicker patients may die more quickly, allowing inadequate time for their physician to enter relevant comorbidity data. The very process of working up and listing a patient makes it less likely that data will be missing. It is therefore perhaps not surprising that patients activated on the national kidney transplant waiting list are more likely to have ethnicity and PRD data reported $(\mathrm{p}<0.0001)$ (table 9.1).

\section{Centre variation}

The analyses performed within this report highlight significant centre effect in relation to the proportion of patients wait listed with nearly $20 \%$ of centres lying outside the lower $95 \%$ confidence interval, and three centres outside the lower $99.8 \%$ confidence interval, despite adjusting for a range of patient characteristics. Intercentre differences are also noted in access to transplants from donors after cardiac death/living kidney donors with nine centres lying outside the lower $99.8 \%$ confidence interval.

Whilst both these outcomes are subject to individual centre practices and policies (which thus could be deemed a cause of the observed variation), one needs to interpret these results with caution as this study is limited by the lack comprehensive comorbidity data on all patients. Centres with higher prevalence rates of comorbidities would be expected to list proportionally fewer patients to reflect the fact that fewer patients are fit for transplantation. Additionally, it may take longer to activate patients in these centres due to the need for more intensive investigation and medical optimisation prior to transplantation. Indeed lack of comorbidity data limits definitive adjustment for case mix. Other patient level factors which this study too fails to adjust for include social deprivation which has been associated with reduced access to transplantation of a range of organs, as well as the impact of primary renal diagnoses (other than diabetes), health literacy and HLA sensitisation. Also, this study has not analysed the interplay between factors such as social deprivation and ethnicity and whether the observed differences based on ethnicity are likely to persist after adjustment for social deprivation and varying comorbidity burden in different ethnic groups. In essence, the available dataset does not permit definitive adjustment for case mix.

The observation that a patient starting dialysis in a non-transplanting renal centre was less likely to be registered for transplantation or receive a transplant from a donor after cardiac death (or a living kidney donor) compared with patients managed in transplanting renal centres, is interesting as this raises the question of whether patients are being disadvantaged by their address, and if indeed a 'post-code lottery' does exist. Drawing conclusions on this having not fully adjusted for the aforementioned potential confounders is again difficult, although it does add weight to the argument to conduct a more detailed study. Once registered for kidney transplantation, patients in both transplanting and non-transplanting renal centres had an equal chance of receiving a transplant from a donor after brainstem death. This is reassuring as organ allocation is subject to the national allocation algorithm which one would expect to allocate organs equitably.

The UKRR is collaborating with other researchers in the National Institute for Health Research (NIHR) funded Access to Transplant and Transplant Outcome Measures (ATTOM) research project to study access to kidney transplantation in greater detail. ATTOM is a non-interventional, prospective, cohort study that aims to recruit all patients aged 18-75 years starting dialysis, receiving a transplant and a similar number of matched patients active on the transplant waiting list, from all dialysis and transplant centres in the UK over a one year period. It is hoped that this study will provide greater insight into the barriers in access to transplantation, and that accurate comprehensive comorbidity data collected as part of this study will allow for more accurate adjustment for case mix for future analyses, and will hopefully more accurately demonstrate whether true inter-centre variation exists. This study will also allow practices identified in the better performing centres to be disseminated to other centres, thereby facilitating equity of access to transplantation across the UK.

\section{Conclusions}

This study highlights the persistence of significant centre variation in access to transplantation with respect to the proportion of patients listed and the time taken to activate suitable patients, even after correction for available relevant patient related variables. Significant differences exist between transplanting and nontransplanting centres, with increasing age and diabetes showing a negative association in terms of accessing the transplant wait list. Ethnicity was not seen to affect access to the wait list though did affect the probability of receiving a transplant once listed.

Conflicts of interest: none 


\section{References}

$>1$ Wolfe RA, Ashby VB, Milford EL, Ojo AO, Ettenger RE, Agodoa LY, Held PJ, Port FK. Comparison of mortality in all patients on dialysis, patients on dialysis awaiting transplantation, and recipients of a first cadaveric transplant. N Engl J Medicine 1999;341(23):1725-30

2 Pinson CW, Feurer ID, Payne JL, Wise PE, Shockley S, Speroff T. Health related quality of life after different types of solid organ transplantation. Ann Surg 2000;232(4):597-607

3 Sureshkumar KK, Patel BM, Markatos A, Nghiem DD, Marcus RJ. Quality of life after organ transplantation in type 1 diabetes with end stage renal disease. Clin Transplant 2006;20(1):19-25

4 Garg PP, Furth SL, Fivush BA, Powe NR. Impact of gender on access to the renal transplant waiting list for pediatric and adult patients. J Am Soc Nephrol 2000;11:958-64

5 Alexander GC, Seghal AR. Barriers to cadaveric renal transplantation among blacks, women, and the poor. JAMA 1998;280:1148-52

-6 Gaylin DS, Held PJ, Port FK, Hunsicker LG, Wolfe RA, Kahan BD, et al. The impact of comorbid and sociodemographic factors on access to renal transplantation. JAMA 1993;269:603-8

7 Axelrod DA, Guidinger MK, Finlayson S, Schaubel DE, Goodman DC, Chobanian $\mathrm{M}$, et al. Rates of solid-organ wait-listing, transplantation, and survival among residents of rural and urban areas. JAMA 2008; 299:202-7
8 Sequist TD, Narva AS, Stiles SK, Karp SK, Cass A, Ayanian JZ. Access to renal transplantation among American Indians and Hispanics. Am J Kidney Dis 2004;44:344-52

-9 Wolfe RA, Ashby VB, Milford EL, Bloembergen WE, Agodoa LY, Held PJ, et al. Differences in access to cadaveric renal transplantation in the United States. Am J Kidney Dis 2000;36:1025-33

10 http://www.uktransplant.org.uk/ukt/about_transplants/organ_allocation/ kidney_(renal)/kidney_(renal).jsp

11 Variation between centres in access to renal transplantation in UK: longitudinal cohort study. Ravanan R, Udayaraj U, Ansell D, Collett D, Johnson R, O’Neill J, Tomson CR, Dudley CR. BMJ. 2010 Jul 20; 341:c3451. doi: 10.1136/bmj.c3451

12 Centre variation in access to renal transplantation in the UK (20042006), Ravanan R, O’Neill J, Webb L, Casula A, Johnson R. Clin Pract. 2011;119(suppl 2):c239-48. doi: 10.1159/000331781

13 Social deprivation, ethnicity, and access to the deceased donor kidney transplant waiting list in England and Wales. Udayaraj U, Ben-Shlomo Y, Roderick P, Casula A, Dudley C, Johnson R, Collett D, Ansell D, Tomson C, Caskey F. Transplantation. 2010 Aug 15;90(3): 279-85. doi: 10.1097/TP.0b013e3181e346e3 